\title{
Performing Antegrade Selective Cerebral Perfusion Using the AV Cannula: A Novel Approach
}

\author{
Andrea Venturini, MD, $\mathrm{PhD}^{1}$ (1) Alan Gallingani, $\mathrm{MD}^{1}$ (1) Angiolino Asta, $\mathrm{MD}^{1}$ (1) \\ Chiara Zanchettin, MD ${ }^{1}$ Giampaolo Zoffoli, MD ${ }^{1}$ Antonio Cannarella, MD ${ }^{1}$ \\ Domenico Mangino, $\mathrm{MD}^{1}$
}

${ }^{1}$ Department of Cardiac Surgery, Ospedale dell'Angelo, Mestre,

Address for correspondence Alan Gallingani, MD, Department of Venezia, Italy Cardiac Surgery, Ospedale dell'Angelo, Via Paccagnella,11, Mestre, AORTA 2019;7:181-184. Venezia 30174, Italy (e-mail: gallingalan@gmail.com).

\begin{abstract}
Antegrade selective cerebral perfusion has become the preferred choice for brain protection during aortic arch surgery. To perform antegrade selective cerebral perfusion, cannulas have been introduced directly into the ostia of the supra-aortic vessels (SAV) after institution of hypothermic circulatory arrest and opening the aortic arch. We describe a different surgical technique with a new type of cannula for antegrade selective cerebral perfusion. This cannula, called AV (Andrea Venturini) cannula, has

Keywords

- cerebral protection

- aortic arch

- aortic dissection

- antegrade cerebral perfusion been designed to be introduced in the SAV directly using a standard guidewire technique (Seldinger's technique). The AV cannula can also be introduced from the ostia of the SAV if preferred. The AV cannula can be introduced before the institution of hypothermic circulatory arrest and before opening the aortic arch. One great advantage of this technique is that the ostia of the SAV remain free from a cannula, allowing the operator easier access and a faster anastomosis or reimplantation.
\end{abstract}

\section{Introduction}

Brain protection is one of the crucial issues in aortic arch surgery. Possible techniques include deep hypothermic circulatory arrest, retrograde cerebral perfusion, or the combination of both. ${ }^{1,2}$

Following the first publications by Profs. Kazui et al and Bachet et $\mathrm{al}_{1}{ }^{3-5}$ antegrade selective cerebral perfusion (ASCP) has become the preferred choice in most institutions.

Because the cannula in the original technique had to be placed through the ostia of the supra-aortic vessel (SAV), it needed hypothermic circulatory arrest (HCA) and opening of the aortic arch before instituting ASCP. The brain was not protected during this initial period (opening of the arch), although that is usually very short, in some cases can take a few minutes or substantially more in redo procedures.

The presence of the cannula in the SAV ostia can also get in the way of the operator leading to a more demanding anastomosis with longer circulatory arrest times.

received

February 20, 2018

accepted after revision

November 2, 2019
DOI https://doi.org/

10.1055/s-0039-3401997. ISSN 2325-4637.
The cannulas also have to be removed before the completion of the anastomosis, exposing the brain to further unprotected ischemic time.

We describe a new surgical technique with the AV (Andrea Venturini) cannula (Med Europe SRL, Bologna, Italy) that is introduced directly into the SAV vessel so that the brain perfusion can be commenced immediately before HCA, freeing the SAV ostia for easier access and reimplantation.

\section{Methods}

AV cannula is a silicone cannula with a malleable steel shaft. It has been specifically designed to be introduced directly into SAV and not just from the ostia of the vessel. This cannula is similar to existing cannulas with an inflatable balloon at the tip to seal the vessel proximally while perfusing distally (-Fig. 1).

The novelty of this device is the possibility of a guidewire introduction using the Seldinger's technique. ${ }^{6}$

Copyright $\odot 2019$ by Thieme Medical Publishers, Inc., 333 Seventh Avenue, New York, NY 10001, USA. Tel: +1(212) 760-0888.
License terms

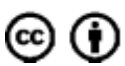




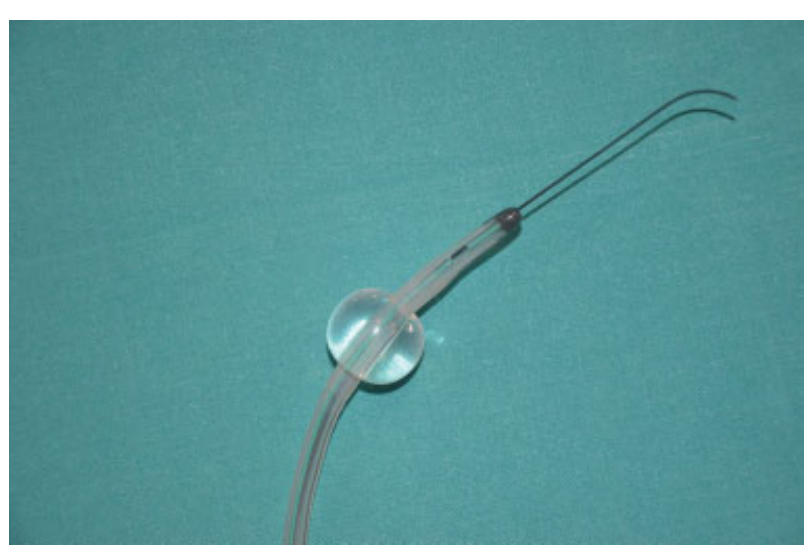

Fig. 1 AV cannula with a guidewire. AV, Andrea Venturini.

After median sternotomy and institution of cardiopulmonary bypass, SAV are isolated and encircled with an umbilical tape. We routinely ligate the innominate vein only during total arch replacement surgery.

A 5/0 prolene (possibly pledget reinforced) purse string suture is placed on the innominate artery and on the left common carotid artery wall. The vessel is then punctured with a specific needle and a guidewire is introduced a few centimeters into the lumen of the vessel. If required, before introducing the cannula a small incision with a number 11 blade can also be done on the vessel wall. If necessary, standard available vessel introducing dilators can be used to facilitate this maneuver.

The AV cannula is then inserted into the innominate artery and in the left common carotid artery and a purse string suture is tied and secured (-Fig. 2).

If required the left subclavian artery can also be cannulated with the same devices and technique.

As systemic cardiopulmonary bypass is discontinued, ASCP is started with a flow of 10 to $15 \mathrm{~mL} / \mathrm{kg} / \mathrm{min}$ maintaining NIRS (Near Infrared Spectroscopy) values between 50

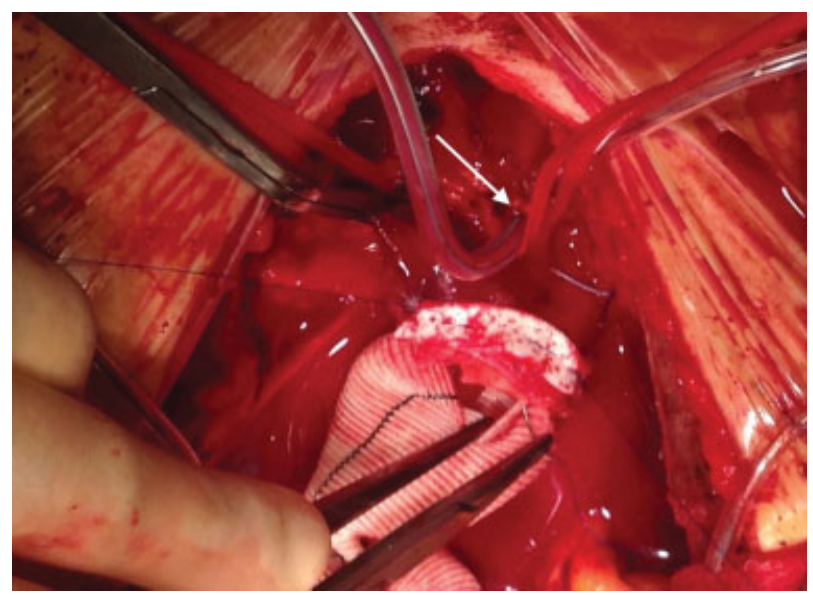

Fig. 2 Andrea Venturini cannula is introduced in to the left common carotid artery (white arrow) during hemiarch operation. A clamp is on the innominate artery for right brain perfusion through a right axillary artery cannula. and $80 \% \mathrm{rsO} 2$, as described in the literature. ${ }^{7-10}$ Blood pressure from the cannula itself can also be monitored.

The required arch operation ${ }^{11}$ is then performed and only after restarting full-flow cardiopulmonary bypass the AV cannulas can be removed.

\section{Results}

We operated two patients so far using the AV cannula. The first one was a 67-year-old male who presented with a typical Type A acute aortic dissection without involvement of the SAV. We performed replacement of the ascending aorta and the proximal arch (hemiarch operation). The second patient was a 74-year-old male with a contained rupture of the dissected proximal descending thoracic aorta and severe mitral valve regurgitation. He previously underwent ascending aorta replacement for acute Type A dissection, subsequently aortic root replacement 1 year later. We performed a total arch replacement with the frozen elephant trunk technique using a Thoraflex Hybrid prosthesis (Vascutek Terumo, Scotland, United Kingdom) and mitral valve replacement using St. Jude Medical mechanical prosthesis (St. Paul, MN).

In the first patient, cardiopulmonary bypass time was 97 minutes, aortic crossclamp time was 84 minutes, circulatory arrest and selective cerebral antegrade perfusion times

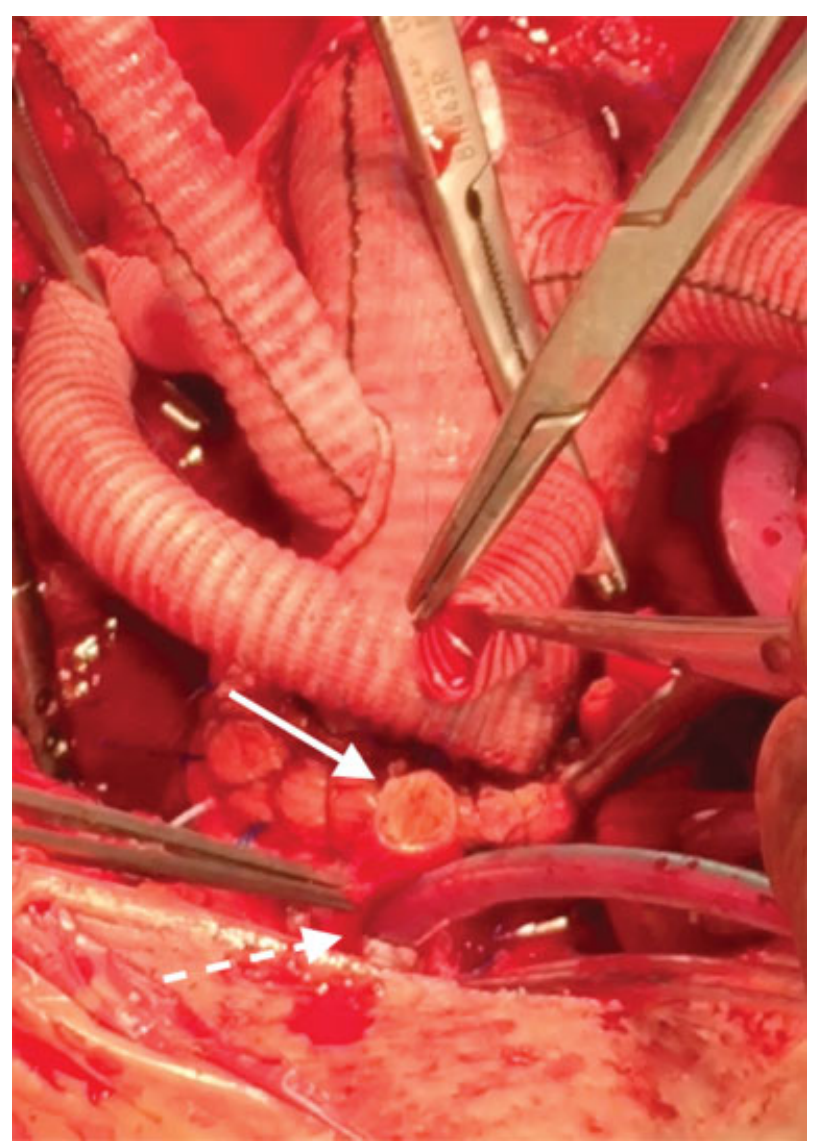

Fig. 3 Left internal carotid artery anastomosis. Free anastomotic site indicated by white arrow; AV cannula insertion site indicated by dashed white arrow. 


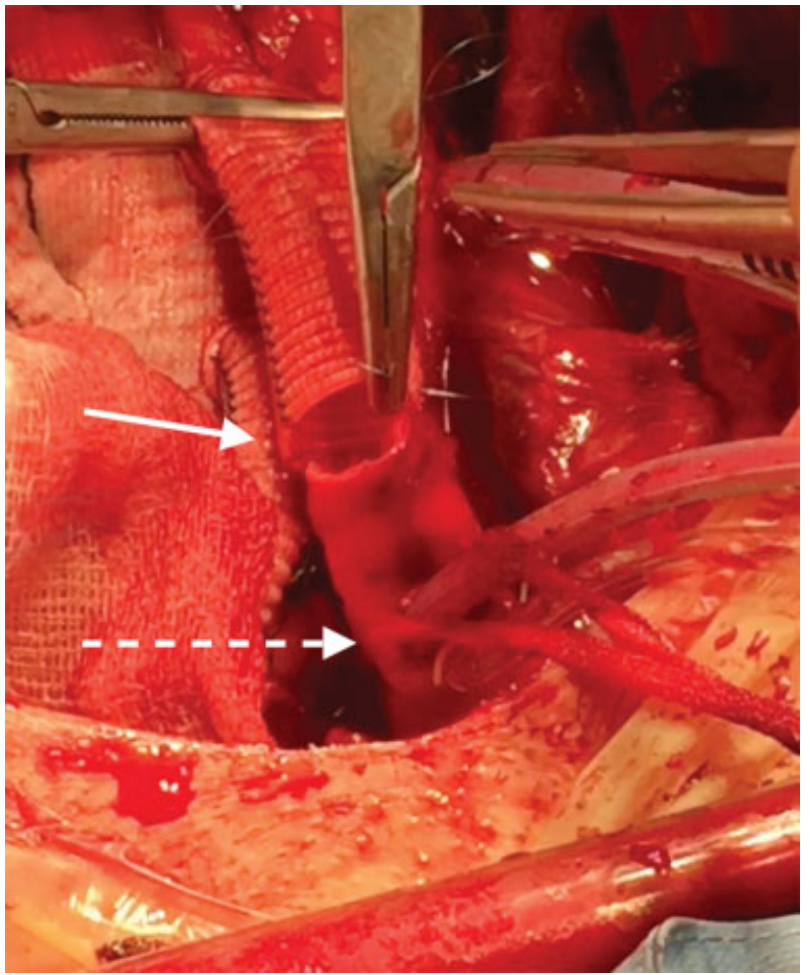

Fig. 4 Innominate artery anastomosis. Free anastomotic site indicated by white arrow; Andrea Venturini cannula insertion site indicated by dashed white arrow.

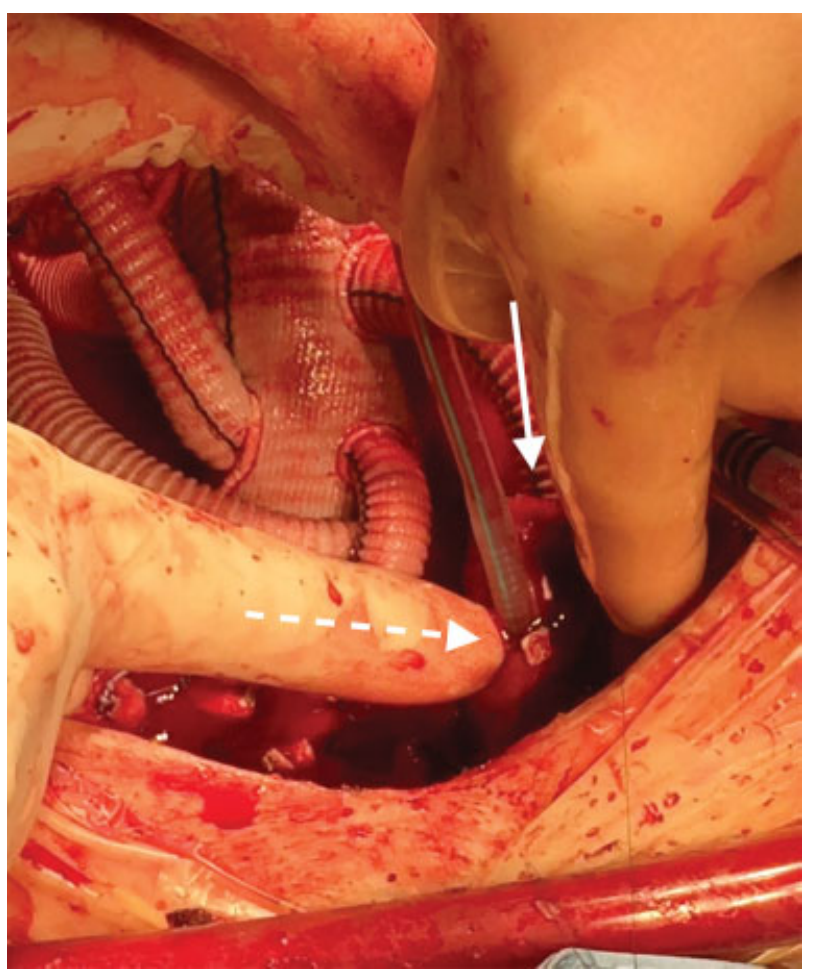

Fig. 5 Andrea Venturini (AV) Cannula removal. Dashed white arrow: easy AV cannula removal, thanks to enough distance from anastomosis line (white arrow).

were 22 minutes; whereas in the second patient, the times were $246,153,44$, and 95 minutes, respectively.

No patient required rethoracotomy for bleeding; furthermore, no stroke and spinal cord injury were detected. At the

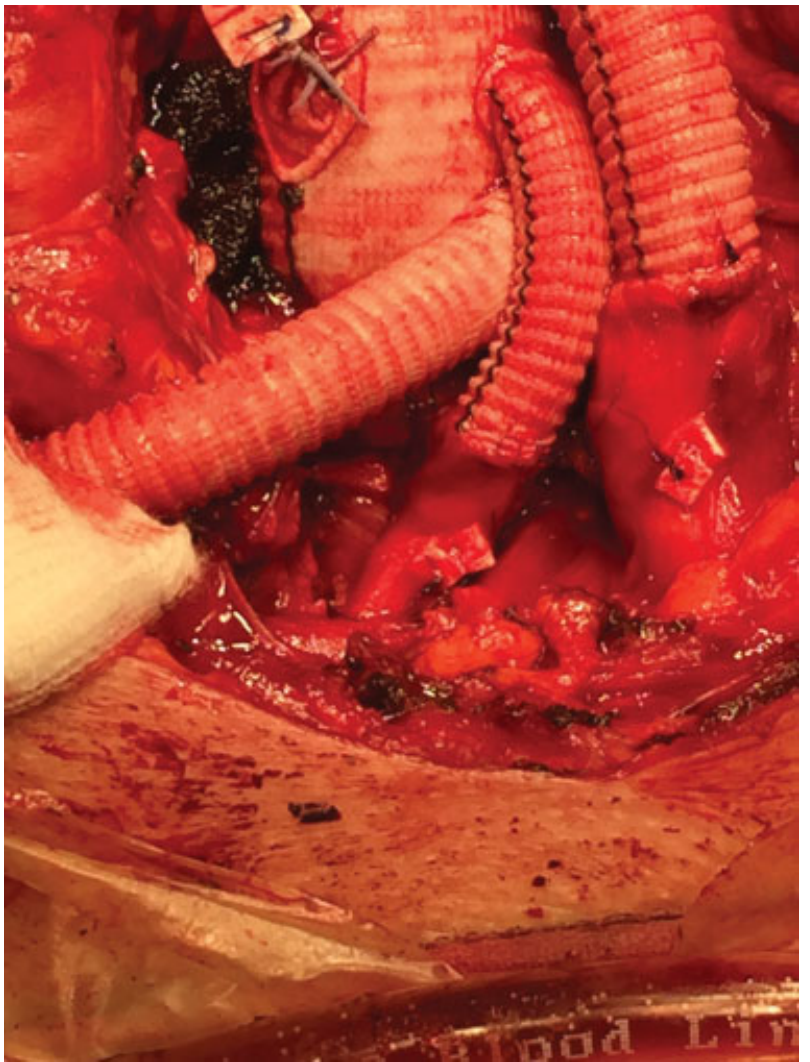

Fig. 6 Supra-aortic vessels detail after frozen elephant trunk operation.

moment, both intraoperatively and at CT scan follow-up no significant stenosis of the cannulation sites were noted. Follow-up at 6 months found that both patients are alive and free of new onset major neurological events.

\section{Conclusion}

Transarterial introduction using Seldinger's technique of the AV cannula represents an alternative to the current wellestablished techniques.

The major advantages of the technique we describe are complete cerebral protection throughout the HCA time and easier arch vessel reimplantation or hemiarch operations as the AV cannula is out of the way of the surgeon (-Figs. 3-6).

The only controindication at present is the presence of dissection of the SAV vessel itself. In this situation, the traditional technique remains the preferred option.

The AV cannula technique for ASCP could possibly be a safer option for instituting cerebral perfusion during HCA in selected patients; in many situations, it is a faster and easier alternative to the existing surgical technique.

Funding

None.

Conflict of Interest

The authors declare no conflict of interest related to this article. 
184 Performing Antegrade Selective Cerebral Perfusion Using AV Cannula Venturini et al.

\section{Acknowledgments}

None.

\section{References}

1 Svensson LG, Crawford ES, Hess KR, et al. Deep hypothermia with circulatory arrest. Determinants of stroke and early mortality in 656 patients. J Thorac Cardiovasc Surg 1993;106(01):19-28, discussion 28-31

2 Ueda Y, Miki S, Kusuhara K, Okita Y, Tahata T, Yamanaka K. Surgical treatment of aneurysm or dissection involving the ascending aorta and aortic arch, utilizing circulatory arrest and retrograde cerebral perfusion. J Cardiovasc Surg (Torino) 1990;31 (05):553-558

3 Kazui T, Inoue N, Komatsu S. Surgical treatment of aneurysms of the transverse aortic arch. J Cardiovasc Surg (Torino) 1989;30 (03):402-406

4 Bachet J, Guilmet D, Goudot B, et al. Cold cerebroplegia. A new technique of cerebral protection during operations on the transverse aortic arch. J Thorac Cardiovasc Surg 1991;102(01):85-93, discussion 93-94

5 Kazui T, Inoue N, Yamada O, Komatsu S. Selective cerebral perfusion during operation for aneurysms of the aortic arch: a reassessment. Ann Thorac Surg 1992;53(01):109-114

6 Seldinger SI. Catheter replacement of the needle in percutaneous arteriography; a new technique. Acta Radiol 1953;39(05):368-376
7 Tanaka H, Kazui T, Sato H, Inoue N, Yamada O, Komatsu S. Experimental study on the optimum flow rate and pressure for selective cerebral perfusion. Ann Thorac Surg 1995;59(03):651-657

8 Di Bartolomeo R, Di Eusanio M, Pacini D, et al. Antegrade selective cerebral perfusion during surgery of the thoracic aorta: risk analysis. Eur J Cardiothorac Surg 2001;19(06):765-770

9 Pacini D, Leone A, Di Marco L, et al. Antegrade selective cerebral perfusion in thoracic aorta surgery: safety of moderate hypothermia. Eur J Cardiothorac Surg 2007;31(04):618-622

10 Misfeld M, Mohr FW, Etz CD. Best strategy for cerebral protection in arch surgery - antegrade selective cerebral perfusion and adequate hypothermia. Ann Cardiothorac Surg 2013;2(03):331-338

11 Bachet J. Open repair techniques in the aortic arch are still superior. Ann Cardiothorac Surg 2018;7(03):328-344

\section{Editor's Questions}

1. Is this new aortic branch vessel perfusion cannula approved
for use in Europe? In the United States?

The new AV cannula has obtained approval for use in Europe, but not yet in the United States. 